\title{
JOINT ESTIMATION OF IMAGE AND FIELDMAP IN PARALLEL MRI USING SINGLE-SHOT ACQUISITIONS
}

\author{
Antonios Matakos and Jeffrey A. Fessler \\ Department of Electrical Engineering and Computer Science \\ The University of Michigan \\ 1301 Beal Avenue, Ann Arbor, MI 48109-2122
}

\begin{abstract}
We propose a method for joint reconstruction of dynamic images and fieldmaps in parallel MRI, using single-shot trajectories. We exploit the sensitivity encoding from parallel imaging to reduce the length of acquisition and essentially perform joint reconstruction using just one full k-space dataset. We also explore the use of modified trajectories (both EPI and spiral) that provide full coverage of k-space and also contain enough inherent time differences to permit accurate fieldmap estimation. Finally we improve the efficiency of the reconstruction algorithm by using a linearization technique for fieldmap estimation, which allows the use of the conjugate gradient algorithm.
\end{abstract}

Index Terms - Parallel MRI, fieldmap estimation, singleshot trajectories, iterative reconstruction

\section{INTRODUCTION}

In functional MRI one reconstructs a series of dynamic images and since high temporal resolution is required it is common to use fast single-shot acquisitions such as echo-planar (EPI) or spirals. The disadvantage of these techniques is the long readout time that can cause significant artifacts in the reconstructed image due to field inhomogeneities if uncorrected. As proposed in [1] one can reconstruct an undistorted image and undistorted dynamic fieldmap using spiralin/spiral-out acquisition. However acquiring two full datasets in a single acquisition may result in very long readout times. In this work, motivated by [2], we propose to use the sensitivity encoding [3] to acquire just one full dataset from a single-shot acquisition (shorter readout time) and still be able to reconstruct both the image and dynamic fieldmap.

Another disadvantage of the method in [1] is that the method used for fieldmap estimation in nonlinear and computationally demanding. The method described in [4] can significantly improve the efficiency of fieldmap estimation since the linearization that is used permits the use of conjugate gradient $(\mathrm{CG})$ which is much more efficient compared to the gradient descent (GD) method used in [1].

This work was supported in part by NIH grant P01 CA87634.
This work proposes a method for jointly estimating the image and dynamic fieldmap in parallel MRI, using a singleshot acquisition that uses just one full dataset. This method retains the advantage of high temporal resolution of singleshot trajectories along with the advantage of shorter readout time by exploiting the coil sensitivity encoding. The shorter readout time has the potential to significantly improve the reconstruction quality since it leads to less distortion due to field inhomogeneity. We also propose the use of modified singleshot trajectories (both EPI and spiral) that provide full coverage of k-space and also allow for enough time differences between regions of $\mathrm{k}$-space so that the fieldmap estimation is facilitated (see $\S 3$ ). Finally, in this work we will use the linearization technique for fieldmap estimation [4] to improve the efficiency of the reconstruction algorithm.

\section{THEORY}

Parallel MRI uses multiple receiver coils and the coil sensitivity patterns provide extra information that we can use for image reconstruction. Assuming that we have $n_{c}$ coils the sensitivity of each coil $c_{i}(r)$ is location dependent and the signal equation for the $i$ th coil at time $t$ is expressed as:

$$
s_{i}(t)=\int c_{i}(r) f(r) e^{-i \omega(r) t} e^{-i 2 \pi k(t) \cdot r} \mathrm{~d} r, i=1, \ldots, n_{c},
$$

where $f(r)$ is the object's magnetization at location $r, \omega(r)$ is the field inhomogeneity and $k(t)$ is the trajectory. By parametrizing the signal equation using basis functions for the image, fieldmap and coil sensitivities (rect basis for now) [1] we have:

$$
s_{i}(t)=\Phi(k(t)) \sum_{n=0}^{N-1} c_{i, n} f_{n} e^{-i \omega_{n} t} e^{-i 2 \pi k(t) \cdot r_{n}},
$$

where $\Phi(k)$ is the Fourier transform of the basis function $\phi(r)$, and $f_{n}, \omega_{n}, c_{i, n}$ denote the pixel values of $f(r), \omega(r)$, $c_{i}(r)$ respectively.

The MRI measurements are noisy samples of this signal:

$$
y_{i, m}=s_{i}\left(t_{m}\right)+\varepsilon_{m}, \text { for } m=1, \ldots, M,
$$


and we can express the noisy measurements $y_{i}$ for each coil in matrix-vector form as follows:

$$
y_{i}=A\left(\omega, c_{i}\right) f+\varepsilon_{i}, \text { for } i=1, \ldots, n_{c},
$$

where $f$ is the discretized object and the elements of the ma$\operatorname{trix} A\left(\omega, c_{i}\right)$ are

$$
a_{m, n}=\Phi\left(k\left(t_{m}\right)\right) e^{-i \omega_{n} t_{m}} e^{-i 2 \pi k\left(t_{m}\right) \cdot r_{n}} c_{i, n} .
$$

The total measurement vector $y$ is given by stacking the measurement vectors $y_{i}$ for each coil: $y=\left[y_{1}, \ldots, y_{n_{c}}\right]^{T}$. Hence the overall $n_{c} M \times N$ system matrix $A_{C}(\omega)$ is given by stacking the system matrices $A\left(\omega, c_{i}\right)$ for each coil:

$$
A_{C}(\omega)=\left[\begin{array}{c}
A\left(\omega, c_{1}\right) \\
\vdots \\
A\left(\omega, c_{n_{c}}\right)
\end{array}\right] .
$$

Using the above, the overall measurement model in matrixvector form can be written as:

$$
y=A_{C}(\omega) f+\varepsilon .
$$

To estimate $f$ and $\omega$ we have to minimize a cost function similar to the one derived in [1], with the only difference that we use $A_{C}$ as the system matrix:

$$
\Psi(f, \omega)=\frac{1}{2}\left\|y-A_{C}(\omega) f\right\|^{2}+\beta_{1} R(f)+\beta_{2} R(\omega),
$$

where $R(f)$ and $R(\omega)$ are quadratic regularization terms $\|C f\|^{2}$ and $\|C \omega\|^{2}$ respectively, and $C$ is a matrix of second order differences.

We want to jointly estimate $f$ and $\omega$ by minimizing $\Psi$ :

$$
\hat{f}, \hat{\omega}=\arg \min _{f, \omega} \Psi(f, \omega) .
$$

Following the idea from [4] we can solve (3) for $\omega$ using a linear approximation to the dynamic changes between $\omega$ and a carefully chosen reference $\check{\omega}$. By doing that we can avoid using the computationally demanding GD method and use the CG method instead to solve for $\omega$. Typically we obtain $\check{\omega}$ from a prescan or by using the estimate $\hat{\omega}$ from the previous dynamic frame.

Assuming that we have a reliable initial estimate $\breve{\omega}$ we can write the signal equation for each coil (1) as:

$$
s_{i}(t)=\Phi(k(t)) \sum_{n=0}^{N-1} c_{i, n} f_{n} e^{-i \check{\omega}_{n} t} e^{-i\left(\omega_{n}-\check{\omega}_{n}\right) t} e^{-i 2 \pi k(t) \cdot r_{n}} .
$$

Now, if the difference of $\omega$ and $\check{\omega}$ is small we can use the following first-order Taylor approximation:

$$
e^{-i\left(\omega_{n}-\check{\omega}_{n}\right) t} \approx 1-i t\left(\omega_{n}-\check{\omega}_{n}\right),
$$

and then by substituting (5) in (4) and rearranging the terms, the signal equation becomes:

$$
\begin{aligned}
& s_{i}(t) \approx \Phi(k(t)) \sum_{n=0}^{N-1} c_{i, n} f_{n} e^{-i \check{\omega}_{n} t} e^{-i 2 \pi k(t) \cdot r_{n}}- \\
& \quad i(-t) \Phi(k(t)) \sum_{n=0}^{N-1} c_{i, n} f_{n} e^{-i \check{\omega}_{n} t} e^{-i 2 \pi k(t) \cdot r_{n}} \check{\omega}_{n}+ \\
& i(-t) \Phi(k(t)) \sum_{n=0}^{N-1} c_{i, n} f_{n} e^{-i \check{\omega}_{n} t} e^{-i 2 \pi k(t) \cdot r_{n}} \omega_{n} \\
& \quad \text { for } i=1, \ldots, n_{c} .
\end{aligned}
$$

Using the signal equation (6) we can rewrite the measurement model for each coil (2) in matrix-vector form as:

$$
y_{i}=A\left(\check{\omega}, c_{i}\right) f-B\left(\check{\omega}, f, c_{i}\right) \check{\omega}+B\left(\check{\omega}, f, c_{i}\right) \omega+\varepsilon_{i},
$$

where the elements of the $M \times N$ matrices $A\left(\check{\omega}, c_{i}\right)$ and $B\left(\check{\omega}, f, c_{i}\right)$ are:

$$
\begin{aligned}
& a_{m, n}=\Phi\left(k\left(t_{m}\right)\right) e^{-i \check{\omega}_{n} t_{m}} e^{-i 2 \pi k\left(t_{m}\right) \cdot r_{n}} c_{i, n}, \\
& b_{m, n}=i\left(-t_{m}\right) \Phi\left(k\left(t_{m}\right)\right) e^{-i \check{\omega}_{n} t_{m}} e^{-i 2 \pi k\left(t_{m}\right) \cdot r_{n}} f_{n} c_{i, n} .
\end{aligned}
$$

The overall measurement vector $y$ and the matrices $A_{C}$ and $B_{C}$ are given by stacking the measurement vectors $y_{i}$ and system matrices $A\left(\check{\omega}, c_{i}\right)$ and $B\left(\check{\omega}, f, c_{i}\right)$ for each coil. Hence the overall linearized measurement model is:

$$
y=A_{C}(\check{\omega}) f-B_{C}(\check{\omega}, f) \check{\omega}+B_{C}(\check{\omega}, f) \omega+\varepsilon .
$$

Similarly to (3), to estimate the image $f$ and fieldmap $\omega$ we have to minimize the following cost function:

$$
\begin{aligned}
\Psi(f, \omega)= & \frac{1}{2}\left\|y-A_{C}(\check{\omega}) f+B_{C}(\check{\omega}, f) \check{\omega}-B_{C}(\check{\omega}, f) \omega\right\|^{2} \\
& +\beta_{1} R(f)+\beta_{2} R(\omega) .
\end{aligned}
$$

To minimize the cost function (7) we first minimize over $f$ by using an estimate $\check{\omega}$ for the fieldmap and then we use the estimate $\check{f}$ for the image to minimize over $\omega$. When solving for $f$ we must minimize:

$$
\Psi_{1}(f)=\frac{1}{2}\left\|y-A_{C}(\check{\omega}) f\right\|^{2}+\beta_{1} R(f)
$$

and when solving for $\omega$ we must minimize:

$$
\Psi_{2}(\omega)=\frac{1}{2}\left\|\tilde{y}-B_{C}(\check{\omega}, \check{f}) \omega\right\|^{2}+\beta_{2} R(\omega),
$$

where,

$$
\tilde{y} \triangleq y-A_{C}(\check{\omega}) \check{f}+B_{C}(\check{\omega}, \check{f}) \check{\omega} \text {. }
$$

We minimize both $\Psi_{1}$ and $\Psi_{2}$ using the CG-NUFFT method [1] which is reasonably computationally efficient. 


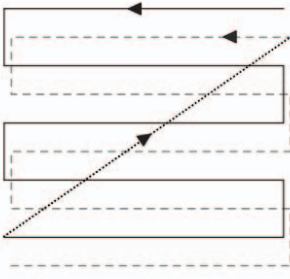

(a) Interleaved EPI trajectory

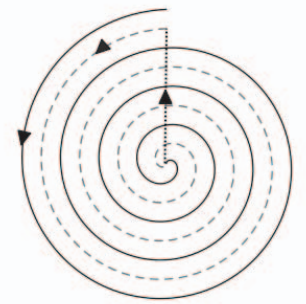

(b) Interleaved spiral-in trajectory
Fig. 1. Single-shot, interleaved trajectories used in simulations.

\section{MATERIALS AND METHODS}

The joint estimation method described in $\S 2$ can be applied with any trajectory, but the results will depend on the chosen trajectory. For parallel imaging (multiple coils) we can exploit the coil sensitivities so that we can achieve joint reconstruction using only one full dataset acquired with a singleshot trajectory [2]. The trajectories we chose are a single-shot "interleaved" EPI (Fig. 1(a)) and a single-shot "interleaved" spiral-in (Fig. 1(b)). These trajectories provide full coverage of the k-space and also they have some time differences between neighboring parts of k-space, which intuitively should facilitate fieldmap estimation. The parameters used for both trajectories are FOV $=22 \mathrm{~cm}$, matrix size $=64 \times 64$. For interleaved EPI we have readout time $=20 \mathrm{~ms}$ and two echo times at $\mathrm{TE}_{1}=5 \mathrm{~ms}$ and $\mathrm{TE}_{2}=15 \mathrm{~ms}$. In this trajectory, since we do not exactly traverse the center of k-space, we define as echo time (TE) the time when we are closest to the center of $\mathrm{k}$-space. For interleaved spiral we have readout time $=16 \mathrm{~ms}$ and two echo times at $\mathrm{TE}_{1}=8 \mathrm{~ms}$ and $\mathrm{TE}_{2}=16 \mathrm{~ms}$.

The necessary matrix-vector multiplications are performed with time segmentation and the use of FFT for EPI trajectories (data are on a cartesian grid) and the use of NUFFT for the spiral trajectories [5]. Time segmentation was performed with $L=8$ time segments and the NUFFT used a $6 \times 6$ interpolation neighborhood with minmax interpolation and two times oversampling for the FFT.

The regularization parameters $\beta_{1}$ and $\beta_{2}$ in (8) and (9) were chosen to achieve a specific spatial resolution [6]. For the image reconstruction we chose the parameter $\beta_{1}$ so that the FWHM of the PSF was 1.2 pixels for EPI and 2 pixels for spiral and for the fieldmap reconstruction we chose the parameter $\beta_{2}$ so that the FWHM of the PSF was 1.7 pixels for both trajectories.

To jointly estimate the image and fieldmap we used the CG method alternating between updating the image and then updating the fieldmap using in total 20 updates. In each update for image or fieldmap we used 15 iterations of the CG method. For parallel imaging, in our simulations, we used

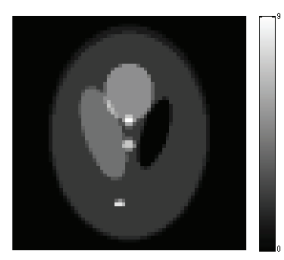

(a) True image

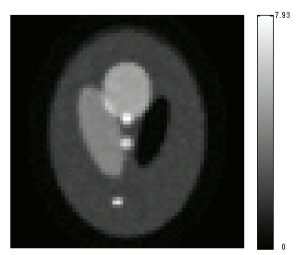

(c) Oracle image estimate

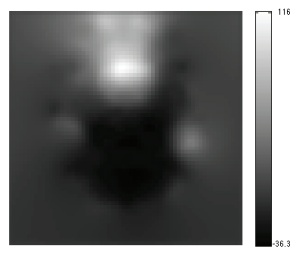

(b) True fieldmap

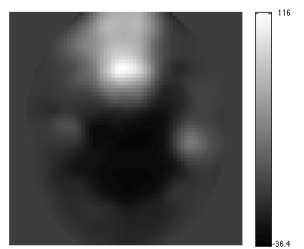

(d) Oracle fieldmap estimate
Fig. 2. True image and fieldmap and oracle estimates for image and fieldmap.

four coils with smooth B1 maps.

\subsection{Simulation}

For the simulations we performed we chose the Shepp-Logan phantom as the true image (Fig. 2(a)) and a smoothed, rapidchanging susceptibility induced, fieldmap acquired from human brain data as the true fieldmap (Fig. 2(b)). The range of the fieldmap is from -36 to $116 \mathrm{~Hz}$. The experiments were performed with simulated data, created using the exact system model, to which we added noise to make a $30 \mathrm{~dB}$ SNR. For the image reconstruction we used an iteratively reconstructed image, uncorrected for field inhomogeneities, as an initial estimate. For the fieldmap reconstruction we used a smoothed standard estimate, from two single-shot EPI acquisitions, as the initial estimate. To further evaluate the quality of the joint reconstruction we created an oracle image estimate that was reconstructed with our method using the true fieldmap (Fig. 2(c)) and we also created an oracle fieldmap estimate that was reconstructed with our method using the true image (Fig. 2(d)). These oracle estimates provide an upper bound on the accuracy of the proposed joint reconstruction method.

\section{SIMULATION RESULTS}

Fig. 3(a) shows the reconstructed image when we do not correct for field inhomogeneities. Because of the fieldmap strength and the long readout time there are significant artifacts. Fig. 3(b) shows the reconstructed image when we correct for field inhomogeneities using the initial, standard fieldmap estimate (Fig. 3(c)). In this case the artifacts are reduced but not completely eliminated and the reconstruction 


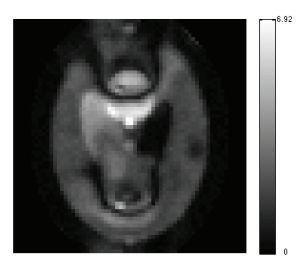

(a) Uncorrected image.
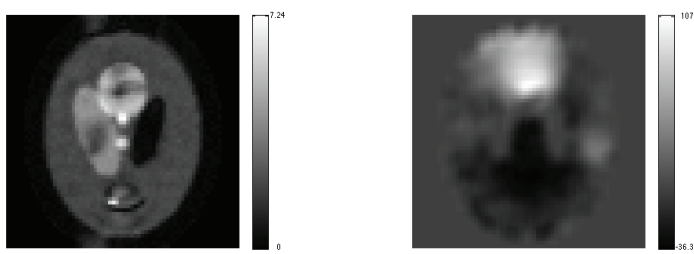

(b) Reconstructed image using ini- (c) Standard (initial) fieldmap estitial fieldmap estimate. mate.

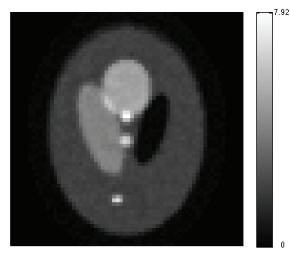

(d) Jointly reconstructed image.

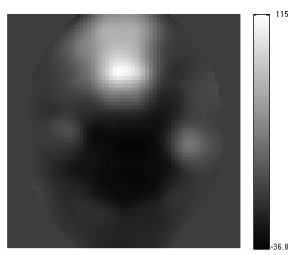

(e) Jointly reconstructed fieldmap.
Fig. 3. Results of reconstruction methods for image and fieldmap using single-shot "interleaved” EPI trajectory.

quality is not close to the one achieved in the oracle reconstruction (Fig. 2(c)) when the true fieldmap is used. This is also evident in terms of normalized RMS error, as seen in Table 1. Fig. 3(d) and 3(e) show the jointly reconstructed image and fieldmap that were created with our proposed method. In this case there are no field inhomogeneity artifacts in the reconstructed image and both the image and fieldmap are very close to the oracle reconstructions (Fig. 2(c) and 2(d)). This can be also seen in terms of normalized RMS error in Table 1. These reconstruction results were created using the single-shot "interleaved" EPI trajectory, but the results for the "interleaved" spiral are also very similar (see Table 1). From these preliminary simulation results we can see that our method seems promising in performing efficient joint reconstruction of image and dynamic fieldmap.

\section{DISCUSSION}

This paper proposed an efficient method for jointly estimating image and fieldmap in parallel MRI. The preliminary simulation results showed that we can achieve high quality reconstruction with this method by using significantly re-

\begin{tabular}{|l|c|c|}
\hline & EPI & Spiral \\
\hline Reconstructed image & $107.5 \%$ & $62.2 \%$ \\
\hline uncorrected & $63.3 \%$ & $35.3 \%$ \\
\hline using initial fieldmap & $8.6 \%$ & $13.7 \%$ \\
\hline oracle (using true fieldmap) & $11.2 \%$ & $14.1 \%$ \\
\hline joint estimation \\
\hline Reconstructed fieldmap \\
\hline standard estimate* & $47.4 \%$ & $47.4 \%$ \\
\hline oracle (using true image) & $2.2 \%$ & $4.0 \%$ \\
\hline joint estimation & $5.5 \%$ & $4.6 \%$ \\
\hline
\end{tabular}

Table 1. Comparative table of NRMS error of reconstruction methods. *Note: The same standard estimate was used for both trajectories as initial fieldmap for joint estimation.

duced datasets compared to standard methods for image and fieldmap estimation. Due to this fact, this method has the potential to be used in functional MRI where dynamic updates of the image and fieldmap are desirable. A disadvantage of this method is that non-standard single-shot trajectories seem to be required to achieve good reconstruction. It would be interesting as a future step to find an analytic relation between trajectories and reconstruction quality as this could explain why the standard trajectories failed to perform well in our simulations. Finally, to further evaluate the proposed method it is necessary to perform experiments using real data from human studies.

\section{REFERENCES}

[1] B. P. Sutton, D. C. Noll, and J. A. Fessler, "Dynamic field map estimation using a spiral-in / spiral-out acquisition," Mag. Res. Med. 51, pp. 1194-204, June 2004.

[2] U. Techavipoo, J. Lackey, J. Shi, T. Leist, and S. Lai, "Geometric distortion correction in EPI by phase labeling using sensitivity encoding (PLUS)," in Proc. IEEE Intl. Symp. Biomed. Imag., pp. 556-9, 2008.

[3] K. P. Pruessmann, M. Weiger, M. B. Scheidegger, and P. Boesiger, "SENSE: sensitivity encoding for fast MRI," Mag. Res. Med. 42, pp. 952-62, Nov. 1999.

[4] V. T. Olafsson, D. C. Noll, and J. A. Fessler, "Fast joint reconstruction of dynamic $R_{2}^{*}$ and field maps in functional MRI," IEEE Trans. Med. Imag. 27, pp. 1177-88, Sept. 2008.

[5] J. A. Fessler and B. P. Sutton, "Nonuniform fast Fourier transforms using min-max interpolation," IEEE Trans. Sig. Proc. 51, pp. 560-74, Feb. 2003.

[6] J. A. Fessler and W. L. Rogers, "Spatial resolution properties of penalized-likelihood image reconstruction methods: Spaceinvariant tomographs," IEEE Trans. Im. Proc. 5, pp. 1346-58, Sept. 1996.

Acknowledgment: The authors acknowledge Doug Noll for discussions of fMRI imaging. 